\title{
Efectos de un programa de ejercicio físico Pilates-Aerobic sobre el nivel de capacidad funcional y la calidad de vida relacionada con la salud física y mental en mujeres mayores
}

\author{
Pedro Jesús RUIZ-MONTERO, María Teresa RAMIRO, Tamara RAMIRO SÁNCHEZ y \\ Eduardo GARCÍA MARMOL
}

\author{
Universidad de Granada
}

(Recibido el 24 de Noviembre de 2019, Aceptado el 7 de Mayo de 2020)

\begin{abstract}
RESUMEN: Los objetivos de este estudio fueron analizar las diferencias entre mujeres activas/no-activas en un programa de ejercicio físico, en la capacidad funcional (fuerza muscular del tren superior e inferior y capacidad cardiorrespiratoria) y calidad de vida relacionada con la salud (función física y salud mental), así como analizar la asociación entre las variables mencionadas y determinar si la capacidad funcional e índice de masa corporal (IMC) son mediadores en la relación entre función física y salud mental. La muestra estuvo compuesta por 340 mujeres $\left(M_{\text {edad }}=69.2 ; D T=6.2\right), 183$ pertenecieron al grupo "activas" en un programa de intervención de ejercicio físico Pilates-Aerobic $\left(M_{\text {edad }}=68.8 ; D T=5.3\right)$, mientras que 157 pertenecieron al grupo "no-activas" $\left(M_{\text {edad }}=69.9 ; D T=7.1\right)$, ya que no recibieron ningún tipo de intervención. Los resultados ponen de manifiesto que las mujeres activas presentan mejor capacidad física funcional y perciben una mejor función física que las mujeres no-activas. La función física y la salud mental, en ambos grupos de mujeres, presentan una correlación negativa. Las variables evaluadas objetivamente en relación a la capacidad funcional física y el IMC no resultaron mediadores en la relación entre función física y salud mental, excepto en el grupo de activas, en las cuales, la fuerza muscular del tren superior es un mediador que potencia la relación negativa entre la percepción de la calidad de vida con respecto a la función física y la salud mental. Se destaca la necesidad de futuras líneas de investigación que permitan discernir cómo el ejercicio físico favorece el estado psicológico en mujeres mayores teniendo en cuenta las recomendaciones sobre el tipo, la frecuencia, el volumen, la densidad y la intensidad de ejercicio físico.
\end{abstract}

Palabras clave: capacidad funcional, calidad de vida relacionada con la salud, mujeres mayores, programa intervención en ejercicio físico

\section{Pilates-Aerobic exercise program's effects in physical fitness level and quality of life related to physical and mental health in elderly women}

ABSTRACT: The objectives of this study were to analyze the differences between women active/ non-active in a physical exercise program, functional capacity (upper and lower body strength and 
cardiorespiratory fitness) and health-related quality of life (physical function and mental health), as well as analyzing the association between the mentioned variables and determining if the functional capacity and body mass index (BMI) are mediators in the relationship between physical function and mental health. The sample consisted of 340 women $\left(M_{\mathrm{age}}=69.2 ; S D=6.2,183\right.$ belonged to the group "active" in a Pilates-Aerobic exercise program $\left(M_{\text {age }}=68.8 ; S D=5.3\right)$, while 157 belonged to the "non-active" group $\left(M_{a g e}=69.9 ; S D=7.1\right)$, since they did not receive program. The results show that the active women have a better functional physical capacity and perceive a better physical function than the non-active women. Physical function and mental health, in both groups of women, present a negative correlation. The variables evaluated objectively in relation to the physical functional capacity and the BMI did not mediate the relationship between physical function and mental health, except in the group of active women, in which the upper body strength is a mediator that enhances the negative relationship between the perception of quality of life with respect to physical function and mental health. The need for future lines of research to discern how physical exercise favors the psychological state in older women taking into account the recommendations on the type, the frequency, workload and intensity of physical exercise is highlighted.

Keywords: Functional Capacity; Health-Related Quality of Life; Older Women; Intervention Program In Physical Exercise.

Correspondencia: E-mail: tramiro@ugr.es

\section{Introducción}

El número de personas mayores de 65 años ha experimentado un crecimiento muy rápido en la población española durante las últimas décadas. Al concluir el año censal 2011, España contaba con 47,190,423 habitantes siendo, porcentualmente hablando, el país del mundo con menor número de jóvenes junto a Japón (Corona-Verdú y Alameda-Gadea, 2018).

En la actualidad en España el 19.16\% de la población se sitúa por encima de los 65 años, un porcentaje que alcanza el $21.46 \%$ si se atiende únicamente a la población femenina de la tercera edad (Banco Mundial, 2015). Si bien es cierto que entre las mujeres españolas de más de 65 años la esperanza de vida es superior a la de los varones, su esperanza de vida saludable resulta ser significativamente menor a causa de una mayor morbilidad (Robine y Beluche, 2015).

La calidad de vida es un objetivo en salud que está adquiriendo cada vez más importancia (Fortuno, 2011) y ha comenzado a utilizarse como medida de resultados en salud (Badia y del Llano, 2000). Asimismo, la Organización Mundial de la Salud (OMS) se ha implicado en implantar un modelo de envejecimiento activo definido en un proceso de optimización de las oportunidades de salud, participación y seguridad con el fin de mejorar la calidad de vida de las personas que están envejeciendo (OMS, 2002). Así, la calidad de vida se define como: "la percepción que cada individuo tiene de su posición en la vida en el contexto del sistema cultural y de valores en que vive y en relación con sus metas, expectativas, estándares y preocupaciones. Éste es un concepto amplio, del que forman parte elementos como la salud física, el estado psicológico, el nivel de independencia, las relaciones sociales y su relación con el ambiente que le rodea" (WHOQOL Group, 1998, p. 1570).

En relación con la salud física y mental, el beneficio de la práctica regular de algún tipo de actividad física y de los riesgos derivados de un estilo de vida sedentario han sido objeto de investigación en numerosos estudios (Bennett, Clarke, Kowalski y Crocker, 2017; Ruiz-Mon- 
tero, Ruiz-Rico Ruiz, Martín-Moya y González-Matarín, 2019; Varoa y Martínez-González, 2007). La evidencia científica demuestra que la realización de actividad física regular y apropiada se configura como el medio más eficaz para fomentar la salud y el bienestar de las personas (Pérez, 2014; Rodríguez, Molina, Jiménez, y Pinzón, 2011). Además, numerosos estudios han evidenciado la asociación entre una menor percepción de calidad de vida relacionada con la salud y una baja capacidad funcional por METs (unidades metabólicas que representan el consumo de oxigeno) o bajo nivel de actividad física (Bassuk y Manson, 2005; Bize, Johnson, y Plotnikoff, 2007).

Respecto a la salud física en la población mayor, una de las manifestaciones más características relacionada a un estilo de vida sedentario es la sarcopenia. Esta disminución de masa muscular está directamente relacionada con la pérdida de fuerza, disminución de la capacidad funcional y empeoramiento de la calidad de vida relacionada con la salud (Goodpaster et al., 2006). Según diversos estudios (Amin et al., 2018; Warner, Roberts, Jeanblanc y Adams, 2017), el ejercicio físico tiene un efecto positivo sobre la salud física y mental debido a que produce liberación de endorfinas y esto, conlleva a una reducción de la ansiedad, la depresión y el estrés. Ya sea jercicio físico de bajo o alta intensidad, la liberación de estas sustancias actúan directamente sobre el cerebro, produciéndose una sensación de bienestar y relajación inmediata. Además, inhiben las fibras nerviosas que transmiten el dolor, generando analgesia y sedación (Arruza et al., 2008). Todo ello produce un crecimiento de la autonomía personal y mayor autoestima en la población mayor que realiza ejercicio físico regular (Marshall, Schabrun, y Knox, 2017; Warner et al., 2017).

Por consiguiente, el presente estudio tiene como primer objetivo analizar las diferencias entre las mujeres participantes en un programa de ejercicio físico dirigido (de aquí en adelante conocidas como activas) y mujeres no participantes en dicho programa (de aquí en adelante conocidas como no-activas), en términos clínicos de capacidad funcional (fuerza muscular del tren superior e inferior y capacidad cardiorrespiratoria) y calidad de vida relacionada con la salud (función física y salud mental). El segundo objetivo es el análisis de la asociación entre las variables citadas. El tercer objetivo pretende determinar si la fuerza muscular del tren superior e inferior, la capacidad cardiorrespiratoria e índice de masa corporal (IMC) son mediadores en la relación entre función física y salud mental (Figura 1) de mujeres mayores activas y no-activas en un programa de ejercicio físico dirigido en la provincia de Málaga, al sur de España. 


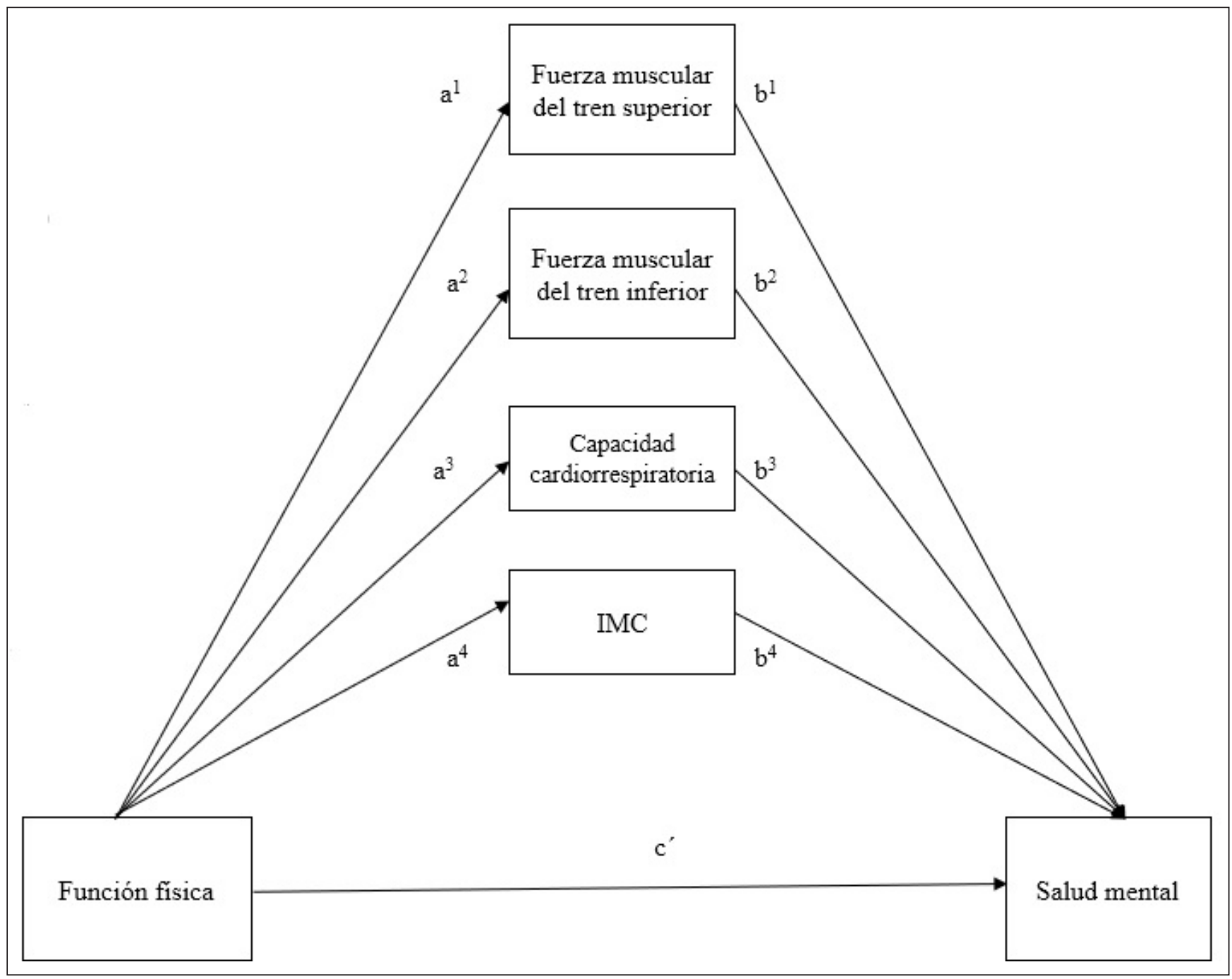

Figura 1. Modelo hipotético de relación entre Función física y Salud mental en activas y no-activas en el presente estudio. a= Vía indirecta entre Función física y cada uno de los cuatro mediadores; $b=$ Vía indirecta entre cada uno de los cuatro mediadores y Salud mental; c'= Vía directa de relación entre Función física y Salud Mental. IMC=Índice de masa corporal.

\section{Método}

\section{Diseño}

Se trata de un diseño cuasi experimental y de corte transversal, con medidas solo post-tratamiento, con dos grupos, uno de cuasi control (Montero y León, 2007).

\section{Participantes}

La muestra estuvo compuesta por 340 mujeres $\left(M_{\text {edad }}=69.2 ; D T=6.2\right)$, de las cuales 183 pertenecieron al grupo "activas" en un programa de intervención de ejercicio físico Pilates-Aerobic, mayores de 60 años $\left(M_{\text {edad }}=68.8 ; D T=5.3\right)$, mientras que 157 pertenecieron al grupo "no-activas" 
$\left(M_{\text {edad }}=69.9 ; D T=7.1\right)$, ya que no recibieron ningún tipo de programa dirigido de ejercicio físico. Todas las mujeres incluidas en el estudio residían en tres poblaciones ubicadas geográficamente en el área rural conocido como Sierra de las Nieves (Málaga), concretamente en poblaciones menores de 2,500 habitantes.

\section{Instrumentos}

Características clínicas. Se elaboró un cuestionario ad hoc, para conocer las características clínicas de la muestra y el número de enfermedades que habían padecido.

Composición corporal. Se utilizó una báscula TANITA BC-601 con la que se registró el Índice de Masa Corporal $\left(\mathrm{Kg} / \mathrm{m}^{2}\right)$. La talla $(\mathrm{cm})$ fue medida usando un estadiómetro (Seca 22, Hamburg, Germany).

Calidad de vida relacionada con la salud. Se utilizó la versión Española del SF-36 Health Survey (Alonso, Prieto, y Anto, 1995)performed within the International Quality of Life Assessment project (including researchers from 15 countries. Este cuestionario se compone de 36 ítems, agrupados en dos componentes de cuatro dimensiones cada una (ocho en total): componente físico (Función física, Rol físico, Dolor corporal y Salud general) y componente mental (Vitalidad, Función social, Rol emocional y Salud mental). Para el presente estudio solo se ha tenido en cuenta la dimensión de Función física (10 ítems) y Salud mental (5 ítems). Cada una de las ocho dimensiones que conforman este cuestionario tiene un rango de puntuación que oscila entre 0 y 100 , donde 0 indica el peor estatus posible de salud y 100 el mejor estatus posible.

Nivel de capacidad funcional. Se empleó el Senior Fitness Test (SFT, Rikli y Jones, 2001) Second Edition, $\backslash$ " offers the most comprehensive and reliable test battery for assessing physical fitness in adults ages 60 and older. The tests in this resource assist health practitioners and fitness and rehabilitation specialists in identifying weaknesses that cause mobility problems, developing exercise programs that improve functional fitness, preventing future mobility issues, and evaluating progress. The Senior Fitness Test (SFT, que evalúa la fuerza de tren superior e inferior y la capacidad cardiorrespiratoria. La fuerza del tren inferior (30-s chair stand), se evalúa a través del número de veces que una participante puede levantarse completamente desde una posición sentada, durante 30 segundos y con los brazos en cruz pegados al pecho. La fuerza de tren superior ( arm curl), se evalúa a través de un test realizado con una mancuerna de $2.27 \mathrm{~kg}$ para mujeres. Cada participante realiza (alternativamente con cada brazo) durante 30 segundos todas las flexiones y extensiones de brazo posible. Para evaluar la capacidad cardiorrespiratoria (6-minute-walk), la participante debe de caminar durante seis minutos alrededor de un rectángulo de $18.8 \times 4.57$ metros. Al finalizar la prueba, se mide la cantidad de metros que ha recorrido y se registra el valor para el análisis.

Percepción subjetiva del esfuerzo. Un indicador de control y evaluación de la intensidad y efectos hacia el ejercicio físico es la prueba subjetiva del esfuerzo. La usada en el presente estudio es una de las más conocidas y usadas, en su versión moderna (Borg, 1998). Comúnmente conocida como "escala de Borg", este instrumento se compone de una escala de 0 a 10 puntos con una valoración subjetiva que oscila desde muy, muy suave o reposo total (0 puntos) hasta esfuerzo máximo о тиy, тиy duro. La utilización de este instrumento suele ser individual ya que cada participante puede percibir un nivel de intensidad o esfuerzo realizado de manera muy diferente. 


\section{Procedimiento}

En primer lugar, se contactó con las participantes en el estudio a través de vía telefónica o por contacto directo. Las participantes dieron su consentimiento para participar en el presente estudio de forma voluntaria. Posteriormente, se suministró un cuestionario para verificar si las mujeres mayores eran elegibles siguiendo los criterios de inclusión: no tener enfermedades graves o terminales y no padecer una limitación de movilidad funcional. Después se proporcionó información sobre el proceder del estudio y se solicitó el consentimiento informado.

En siguiente lugar, se elaboraron dos grupos, el grupo denominado "activas" estuvo compuesto por asistentes a un programa concurrente de ejercicio físico de Pilates-Aerobic, pertenecientes al programa de Condición Física de la Diputación Provincial de Málaga. Durante el desarrollo de las sesiones se trabajaron componentes de la condición física tales como la flexibilidad y fuerza tanto del tren inferior como superior y, agilidad, por parte del método Pilates (3-4 ejercicios de 8-12 repeticiones en función de la intensidad de la sesión). Alternándose en la misma sesión, la capacidad cardiorrespiratoria y movimientos aeróbicos fueron abordados mediante ejercicios aeróbicos y método Aerobic (al menos 10 minutos de actividad continua vigorosa). Mientras que los ejercicios de Pilates eran sin base musical y con/sin soporte material (nunca en el suelo por la complejidad de las participantes para tumbarse y levantarse), el Aerobic siempre se desarrollaba con música. Destacar que a veces se podían alternar ejercicios de Pilates con ejercicios aeróbicos, sin que estos últimos fueran acompañados de base musical. La primera parte de cada sesión se enfocaba a la metodología Pilates con el fin de trabajar respiración, tonificación muscular, flexibilidad y agilidad y, aumentar la activación vegetativa. Los ejercicios que implicaban la capacidad aeróbica se desarrollaban en la segunda parte de la sesión a excepción de la parte final de la misma, donde se disminuía la intensidad de los ejercicios. Las actividades propuestas en cada sesión siempre eran individuales con el fin de que el tiempo útil fuese el máximo.

La frecuencia de las sesiones fue de dos veces por semana y con una duración de 60 minutos total pero con un tiempo de actividad útil de 45-50 minutos por sesión (Figura 2). La intervención tuvo una duración de 24 semanas (Ruiz-Montero, Castillo-Rodríguez, Mikalacki, y Delgado-Fernández, 2015) y fue supervisada por especialistas en Ciencias del Deporte tanto en el desarrollo de la actividad grupal como en cualquier necesidad personal de las participantes. Tanto el volumen como la frecuencia de las actividades propuestas en cada sesión siempre respetaron las recomendaciones del Colegio Americano de Medicina Deportiva (siglas en inglés, ACSM) y de la Asociación Americana del Corazón (siglas en inglés, AHA) (Nelson et al., 2007). La densidad de trabajo es intermedia-vigorosa pero acorde a la características de las participantes. El tiempo de activación es de intensidad intermedia pero no así la vuelta a la calma, el descanso intermedio de sesión es corto y tanto el trabajo de Pilates como el de Aerobic/ejercicios aeróbicos es de intensidad alta.

Durante las cuatro primeras semanas se usó una intensidad baja en las sesiones y a partir de la quinta sesión, la intensidad de las sesiones aumentó a una resistencia media. La percepción subjetiva del esfuerzo se evaluó al final de cada sesión mediante una escala de percepción subjetiva de esfuerzo (Borg, 1998) con el fin de determinar la intensidad de cada sesión. Además, un investigador controló diariamente la ausencia de efectos adversos o inconvenientes durante y tras la sesión de ejercicio físico. 


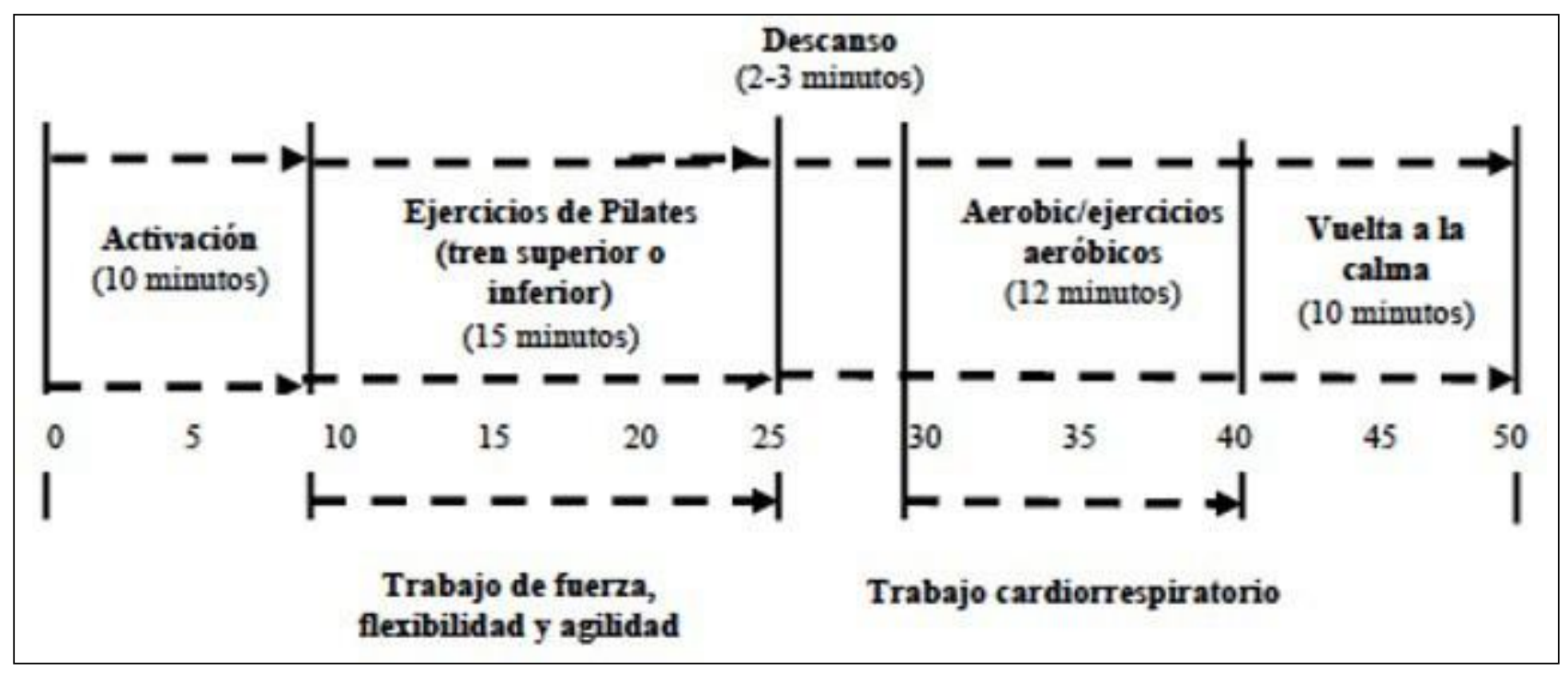

Figura 2. Ejemplo de sesión de entrenamiento concurrente Pilates-Aerobic

El grupo denominado "no-activas" estuvo compuesto por mujeres que eran del mismo contexto geográfico y de iguales características sociodemográficas, pero no asistían a ningún programa de ejercicio físico. Además, semanalmente se contactaba con ellas telefónicamente para comprobar estado de salud y controlar que la práctica de actividad física fuese únicamente la necesaria en actividades y labores diarias.

La evaluación fue realizada, por entrevistadores entrenados, y se han tenido en cuenta los criterios éticos aprobados por el centro público en el que se realizó el estudio y se han respetado los acuerdos de la Declaración de Helsinki. El presente estudio fue aprobado por la Diputación de Málaga y Universidad de Granada.

\section{Análisis estadísticos}

Se ha utilizado la prueba de Kolmogórov-Smirnov para determinar la normalidad de los datos. La determinación de las diferencias significativas entre el grupo de "activas" y "no-activas" fue analizada por una prueba $t$-student en las variables clínicas, capacidad funcional y calidad de vida relacionada con la salud. La magnitud de las diferencias entre grupos fue calculada mediante el tamaño del efecto $\left(\eta_{p}^{2}\right)$, según Cohen (1992). El tamaño del efecto puede ser interpretado como pequeña $(0.2<d<0.5)$, mediano $(0.5<d<0.8)$ o grande $(0.8<d)$.

La asociación de las variables clínicas, capacidad funcional y calidad de vida relacionada con la salud fue analizado a través de una correlación parcial de Pearson. Los valores de correlación para variables basadas en actividad física fueron interpretados como débil o ninguna relación ( $r=0$ a 0.25 ), el grado justo (0.25 a 0.50) y de moderada a buena ( $r=0.50$ a 0.75$)$ (Mannerkorpi, Svantesson y Broberg, 2006).

Respecto a la evaluación de la relación entre función física y salud mental, mediados por las capacidades funcionales de fuerza muscular del tren superior e inferior y capacidad cardiorrespiratoria, además de IMC, se usó un análisis estadístico de mediación múltiple a través de una corrección bootstrap (Preacher y Hayes, 2008). La estimación del bootstrap es un método 
usado para estimar el efecto directo e indirecto en modelos de mediación múltiple. Los intervalos de confianza (IC) de los percentiles bootstrap se analizaron para establecer el tipo de mediación utilizado. Se evidencia falta de significatividad si el valor menor y mayor de IC contiene un cero (Preacher y Hayes, 2008). Además, se comprobó la colinealidad. Todos los análisis estadísticos se realizaron con el paquete estadístico para Ciencias Sociales (IBM SPSS, v.20.0)

\section{Resultados}

La Tabla 1 muestra las diferencias entre características clínicas, capacidad funcional (fuerza muscular del tren superior e inferior y capacidad cardiorrespiratoria) y calidad de vida relacionada con la salud (función física y salud mental) tanto de mujeres mayores "activas" de un programa de ejercicio físico Pilates-Aerobic como de mujeres mayores "no-activas" en ningún tipo de programa físico.

Tabla 1. Diferencias entre Activas y No-Activas del Presente Estudio

\begin{tabular}{|c|c|c|c|c|c|c|c|c|c|}
\hline & & \multirow{2}{*}{\multicolumn{6}{|c|}{$\begin{array}{c}\text { No-activas } \\
(n=157)\end{array}$}} & \multirow{3}{*}{$p$-valor } & \\
\hline & & & & & & & & & \multirow[t]{2}{*}{$\begin{array}{c}\text { Tamaño } \\
\text { del efecto }\end{array}$} \\
\hline & & \multicolumn{6}{|c|}{ Media $\pm D T$} & & \\
\hline \multicolumn{10}{|l|}{ Variables clínicas } \\
\hline Edad & (años) & 68.85 & \pm & 5.37 & 69.92 & \pm & 7.06 & 0.112 & -0.17 \\
\hline IMC & $\left(\mathrm{kg} / \mathrm{m}^{2}\right)$ & 30.48 & \pm & 5.15 & 31.03 & \pm & 4.95 & 0.314 & -0.11 \\
\hline $\begin{array}{l}\text { Número de } \\
\text { enfermedades }\end{array}$ & & 2.31 & \pm & 1.91 & 2.82 & \pm & 2.13 & $0.022^{\mathrm{a}}$ & -0.25 \\
\hline \multicolumn{10}{|c|}{ Capacidad Funcional } \\
\hline $\begin{array}{l}\text { Fuerza muscular } \\
\text { del tren superior }\end{array}$ & ( $\mathrm{n}^{\circ}$ repeticiones) & 18.01 & \pm & 4.14 & 14.42 & \pm & 4.29 & $0.000^{\mathrm{a}}$ & 0.85 \\
\hline $\begin{array}{l}\text { Fuerza muscular } \\
\text { del tren inferior }\end{array}$ & ( $\mathrm{n}^{\circ}$ repeticiones) & 13.27 & \pm & 3.69 & 10.92 & \pm & 3.05 & $0.000^{\mathrm{a}}$ & 0.69 \\
\hline $\begin{array}{l}\text { Capacidad } \\
\text { cardiorrespiratoria }\end{array}$ & (metros) & 451.79 & \pm & 76.91 & 384.11 & \pm & 99.88 & $0.000^{\mathrm{a}}$ & 0.75 \\
\hline \multicolumn{10}{|c|}{ Calidad de vida relacionada con la salud } \\
\hline Función física & $(0-100)$ & 65.76 & \pm & 24.53 & 55.66 & \pm & 24.61 & $0.000^{\mathrm{a}}$ & 0.41 \\
\hline Salud Mental & $(0-100)$ & 36.76 & \pm & 19.93 & 46.22 & \pm & 20.80 & $0.000^{\mathrm{a}}$ & -0.46 \\
\hline
\end{tabular}

Nota. $D T=$ Desviación típica; $I M C=$ Índice de Masa Corporal.

${ }^{\mathrm{a}} p$ valor calculado mediante $t$-student test entre grupo de activas y no-activas para las variables de estudio.

${ }^{\dagger}$ El tamaño del efecto entre activas y no-activas son expresados mediante $d$ de Cohen.

Respecto a la relación establecida entre todas las variables contempladas, los resultados se exponen en la Tabla 2. 
Tabla 2. Correlación entre Variables Clínicas, Capacidad Funcional (Fuerza Muscular del Tren Superior e Inferior y Capacidad Cardiorrespiratoria) y Calidad de Vida Relacionada con la Salud (Función Física y Salud Mental)

\begin{tabular}{lcccccccc} 
& 1 & 2 & 3 & 4 & 5 & 6 & 7 & 8 \\
\hline 1. Edad & & -0.065 & 0.054 & $-0.303^{* *}$ & -0.129 & $-0.508^{* *}$ & $-0.196^{*}$ & -0.086 \\
2. IMC & -0.051 & & 0.090 & -0.008 & 0.103 & $-0.226^{* *}$ & $-0.196^{*}$ & -0.004 \\
3. Número de enfermedades & -0.023 & 0.113 & & -0.076 & 0.013 & $-0.196^{*}$ & $-0.356^{* *}$ & $0.270^{* *}$ \\
4. Fuerza muscular del tren superior & -0.093 & 0.077 & -0.128 & & $0.481^{* *}$ & $0.457^{* *}$ & $0.236^{* *}$ & -0.094 \\
5. Fuerza muscular del tren inferior & -0.051 & -0.082 & -0.095 & $0.556^{* *}$ & & $0.324^{* *}$ & $0.188^{*}$ & -0.150 \\
6. Capacidad cardiorrespiratoria & $-0.312^{* *}$ & $-0.298^{* *}$ & -0.141 & $0.423^{* *}$ & $0.205^{* *}$ & & $0.345^{* *}$ & -0.100 \\
7. Función física & -0.124 & $-0.269^{* *}$ & $-0.370^{* *}$ & $0.214^{* *}$ & $0.170^{*}$ & $0.356^{* *}$ & & $-0.244^{* *}$ \\
8. Salud mental & -0.064 & 0.033 & $0.311^{* *}$ & $-0.273^{* *}$ & $-0.159^{*}$ & -0.127 & $-0.287^{* *}$ & \\
\hline
\end{tabular}

Nota. Coeficientes por encima de la diagonal pertenecen al grupo de activas y por debajo de la diagonal a las no-activas. Ambos grupos mostraron correlaciones significativas al $* p<0.05 ; * * p<0.01$

El análisis de mediación múltiple, usando un bootstrap de 5,000 y sesgo de corrección y aceleración de 95\% del intervalo de confianza (IC) (Preacher y Hayes, 2008), reveló que la ruta desde la función física (x) a los mediadores (m) de fuerza muscular de tren superior e inferior, capacidad cardiorrespiratoria e IMC fueron significativas tanto en el grupo de "activas" como de "no-activas". Sin embargo, la ruta entre los mediadores (m) citados hacia la salud mental (y) no muestra significatividad alguna en ninguno de los dos grupos excepto la fuerza muscular del tren superior en el grupo de "activas" (Tabla 3).

Por consiguiente, el efecto indirecto total de la función física sobre la salud mental a través del mediador fuerza muscular de tren superior fue significativa (punto de estimación=-0.0329, al 95\% IC menor=-0.0834 y mayor=-0.0055). La mediación estimada fue de un $20 \%$ del total del efecto de la función física sobre las mental, mediada por la fuerza muscular de tren superior.

Tabla 3. Efecto Indirecto de la Función Física Sobre la Salud Mental a Través Del IMC y la Capacidad Funcional (Fuerza Muscular del Tren Superior e Inferior y Capacidad Cardiorrespiratoria), ajustada por el número de enfermedades

\begin{tabular}{|c|c|c|c|c|c|c|c|c|}
\hline \multirow{2}{*}{ Mediador } & \multirow{2}{*}{$\begin{array}{c}\text { Efecto de } \mathrm{x} \\
\text { en } \mathrm{m}(a 1-a 4)\end{array}$} & \multirow{2}{*}{$E E$} & \multirow{2}{*}{$\begin{array}{l}\text { Efecto de m } \\
\text { en y }(b 1-b 4)\end{array}$} & \multirow{2}{*}{$E E$} & \multirow{2}{*}{$\begin{array}{l}\text { Estimación } \\
\text { del Bootstrap }\end{array}$} & \multirow{2}{*}{$E E$} & \multicolumn{2}{|c|}{$95 \%$ IC } \\
\hline & & & & & & & Menor & Mayor \\
\hline \multicolumn{9}{|l|}{ Activas $(n=183)$} \\
\hline Fuerza muscular del tren superior & $0.32 *$ & 0.02 & $-1.01 * *$ & 0.33 & -0.0329 & 0.0195 & -0.0834 & -0.0055 \\
\hline Fuerza muscular del tren inferior & $0.03 *$ & 0.01 & -0.57 & 0.37 & -0.0135 & 0.0129 & -0.0523 & 0.0023 \\
\hline Capacidad cardiorrespiratoria & $1.10 * * *$ & 0.23 & -.006 & 0.02 & -0.0075 & 0.0200 & -0.0471 & 0.0332 \\
\hline IMC & $-0.055 * *$ & 0.02 & -0.19 & 0.27 & 0.0109 & 0.0168 & -0.0165 & 0.0519 \\
\hline \multicolumn{9}{|l|}{ No-activas $(n=157)$} \\
\hline Fuerza muscular del tren superior & $0.04 * *$ & 0.02 & -0.19 & 0.38 & -0.0082 & 0.0160 & -0.0501 & 0.0170 \\
\hline
\end{tabular}




\begin{tabular}{|c|c|c|c|c|c|c|c|c|}
\hline \multirow{2}{*}{ Mediador } & \multirow{2}{*}{$\begin{array}{c}\text { Efecto de } \mathrm{x} \\
\text { en } \mathrm{m}(a 1-a 4)\end{array}$} & \multirow{2}{*}{$E E$} & \multirow{2}{*}{$\begin{array}{l}\text { Efecto de } \mathrm{m} \\
\text { en y }(b 1-b 4)\end{array}$} & \multirow{2}{*}{$E E$} & \multirow{2}{*}{$\begin{array}{c}\text { Estimación } \\
\text { del Bootstrap }\end{array}$} & \multirow{2}{*}{$E E$} & \multicolumn{2}{|c|}{$95 \%$ IC } \\
\hline & & & & & & & Menor & Mayor \\
\hline Fuerza muscular del tren inferior & $0.03 * *$ & 0.01 & -0.86 & 0.53 & -0.0237 & 0.0179 & -0.0707 & 0.0003 \\
\hline Capacidad cardiorrespiratoria & $1.28 * * *$ & 0.32 & -0.001 & 0.02 & -0.0002 & 0.0270 & -0.0510 & 0.0573 \\
\hline IMC & $-0.03 *$ & 0.02 & -0.24 & 0.32 & 0.0092 & 0.0182 & -0.0147 & 0.0650 \\
\hline
\end{tabular}

Nota. Basado en una muestra de bootstrap de 5,000. $\mathrm{X}=$ Función física, $\mathrm{Y}=$ Salud mental, $\mathrm{M}=$ mediador, $C=$ Intervalo de confianza, $E E=$ Error Estándar

$\mathrm{Si}$ el IC contiene cero se interpreta como no significativo.

$* p<0.05 ; * * p<0.01 ; * * * p<0.001$

\section{Discusión/Conclusiones}

Los resultados encontrados en el estudio, en relación con el primer objetivo planteado, ponen de manifiesto que las mujeres participantes en un programa de ejercicio físico dirigido presentan menor número de enfermedades y mejor condición física con respecto a la fuerza física del tren superior e inferior, y la capacidad cardiorrespiratoria. Resultados que concuerdan con los obtenidos en otros estudios (Pérez, 2014; Rodríguez et al., 2011) que manifiestan que el ejercicio físico se relaciona positivamente con la salud y la condición física.

En relación con la calidad de vida percibida con respecto a la función física, los resultados concuerdan con los anteriores y las mujeres que realizan ejercicio físico dirigido, perciben una mejor función física. Estos resultados se encuentran en la línea de otros estudios, que han identificado que el ejercicio físico permite a las mujeres mayores ser más independientes mejorando su percepción de bienestar (Costa, 2009) y que las personas sedentarias estaban menos satisfechas que las activas, en relación con su calidad de vida (Capdevilla, 2005). Por el contrario, en contraposición a otros estudios (Silva y Mayán, 2016) son las mujeres que no realizan ningún ejercicio físico dirigido las que perciben una mejor calidad de vida relacionada con la salud mental, cuestión que se analizará más adelante.

Los resultados, en relación con el segundo objetivo, ponen de relieve que existe una relación negativa entre la edad y la capacidad respiratoria en ambos grupos de mujeres, aunque dicha relación es más potente en el grupo de mujeres que practican ejercicio físico dirigido. Además, en el grupo de mujeres que practican ejercicio físico dirigido, se ha evidenciado una correlación moderada entre todas las variables relacionadas con la condición física. Este hecho va en consonancia con estudios previos donde existe una asociación entre la práctica de ejercicio físico dirigido y la mejora de la capacidad funcional en personas mayores (Herrera, Pablos, Chiva-Bartoll, y Pablos, 2017; Phillips, Wójcicki, y McAuley, 2013). Otro estudio sobre trabajo de fuerza y Aerobic ha mostrado los efectos beneficiosos en el proceso de envejecimiento y, especialmente, en la función física mediante un continuo ajuste del volumen de trabajo en cada sesión respecto al volumen de la sesión anterior (Melov, Tarnopolsky, Beckman, Felkey, y Hubbard, 2007). Las cargas de trabajo en cada sesión siempre se adaptaban a la repetición máxima (RM) de cada participante y esto siempre posibilitaba una función física.

Sin embargo, la función física percibida en el presente estudio únicamente se relaciona con la capacidad cardiorrespiratoria, y no con la Fuerza muscular. Además, sólo en el grupo de 
mujeres que no practican ejercicio físico dirigido, aquellas que tienen un mayor IMC presentan menor capacidad cardiorrespiratoria y peor percepción de su función física. Estos resultados se encuentran en la línea de los hallados por Imayama et al. (2013), que demostraron que la percepción de una calidad de vida con respecto a la función física era mayor en sujetos que mostraban mayor adherencia al ejercicio y mayores cambios positivos en la capacidad cardiorrespiratoria.

Con respecto a la función física y la salud mental nos encontramos que, en ambos grupos de mujeres, presentan una correlación negativa, a pesar de ser poco robusta. Así, el hecho de percibir una mejor función física se relaciona con la percepción de una peor salud mental. Según Kanning y Schlitcht (2008) la actividad física promueve un envejecimiento saludable, en primer lugar, por los efectos fisiológicos y cognitivos que produce; y, además, porque las personas pueden satisfacer mejor sus necesidades psicológicas y la percepción de bienestar mientras permanecen físicamente activas. Esta cuestión, igualmente ha sido apoyada por otros estudios llevados a cabo con muestras de mujeres mayores (Silva et al., 2015). Por otro lado, un estudio de ejercicio físico concurrente con volúmenes elevados de trabajo (90 minutos por sesión), densidad media (10 minutos de descanso cada 20 minutos de trabajo físico más múltiples descansos) y ejercicios de fuerza, flexibilidad, Aerobic sumado a un trabajo cognitivo simultáneamente, proporcionó mejoras considerables en la salud mental de personas mayores (Rehfeld et al., 2017). Sin embargo, hay que tener presente que no siempre aquellos grupos de adultos mayores más activos físicamente y con niveles altos de función física presentan una adecuada salud mental (Acree et al.,2006).

Por ello, para estudiar más exhaustivamente dicha relación, se plantea el tercer objetivo del estudio, que fue determinar si las variables evaluadas objetivamente con relación a la capacidad física y el IMC son mediadores en la relación entre función física y salud mental. Los resultados evidencian que dichas variables no son mediadoras en la relación, excepto en el grupo de mujeres activas, en las cuales, la fuerza muscular del tren superior es un mediador que potencia la relación negativa entre la percepción de la calidad de vida con respecto a la función física y la salud mental. En este sentido, Rodrigues et al. (2020) determinan en un estudio con características similares al nuestro y mediante una intervención de ejercicio concurrente de fuerza y trabajo aeróbico con personas mayores, que el trabajo de fuerza tanto de tren inferior como superior produce beneficios en el desarrollo cognitivo en población mayor. En esta línea, se sugiere que el ejercicio físico está estrechamente relacionado con beneficios en el plano tanto cognitivo como social, emocional a nivel general (Stillman, Cohen, Lehman y Erickson, 2016) y de salud mental, específicamente en personas mayores (Ruiz-Montero, Castillo-Rodríguez, Micalacki y Delgado-Fernández, 2015). A todo lo anterior hay que sumar que la práctica de ejercicio físico favorece un mejor estatus psicosocial y contribuye a una mejor función física (Holle et al., 2016) y mental (Lok, Lok y Canbaz, 2017).

Estos resultados pueden estar relacionados con el hecho de que aquellas mujeres que practican ejercicio físico perciben mejor función física, imagen corporal y por consiguiente, son más conscientes de los cambios que se producen en su cuerpo (Ruiz-Montero, Ruiz-Rico Ruiz, Martín-Moya, y González-Matarín, 2019; Seguin, Eldridge, Lynch, y Paul, 2013; Zarshenas, Houshvar, y Tahmasebi, 2013). Una pobre imagen corporal puede provocar baja satisfacción en mujeres mayores y poco interés por la práctica de ejercicio físico durante el tiempo libre (Allender, Cowburn, y Foster, 2006). Sin embargo, una percepción positiva de la imagen corporal es asociada a un mejor equilibrio de la autoestima, mayor calidad de vida o mejor disminución de 
la ansiedad y el estrés (McLean, Paxton, y Wertheim, 2010). Por tanto, las percepciones que se tienen sobre el propio cuerpo y sus alteraciones pueden llegar a ser consideradas como peligrosas, si la actividad física lleva al cuerpo más allá de su capacidad, y lo pone en un estado de estrés provocando situaciones de ansiedad y nerviosismo. Aunque estudios recientes (Gordon y Macdowell, 2017) indican que el entrenamiento de fuerza mejora los síntomas de la ansiedad y depresión, tanto en personas sanas, como en pacientes con patologías mentales o físicas, no se tiene en cuenta muestras de población envejecida, cuestión que habría que tener en cuenta.

Por ello, es necesario llevar a cabo futuras líneas de investigación que permitan discernir cómo el ejercicio físico favorece el estado psicológico en mujeres mayores, teniendo en cuenta las recomendaciones sobre el tipo de ejercicio físico, la frecuencia y la intensidad, que son factores determinantes a tener en cuenta (Nelson et al., 2007).

\section{Referencias}

Acree, L.S., Longfors, J., Fjeldstad, A.S., Fjeldstad, C., Schank, B., Nickel, K.J., y Gardner, A.W. (2006). Physical activity is related to quality of life in older adults. Health and Quality of Life Outcomes, 4, 37-42.

Allender, S., Cowburn, G., y Foster, C. (2006). Understanding participation in sport and physical activity among children and adults: a review of qualitative studies. Health Education Research, 21(6), 826-835.

Alonso, J., Prieto, L., y Anto, J.M. (1995). The Spanish version of the SF-36 Health Survey (the SF-36 health questionnaire): an instrument for measuring clinical results. Medicina Clínica, 104, 771-776.

Amin, F.M., Aristeidou, S., Baraldi, C., Czapinska-Ciepiela, E.K., Ariadni, D.D., Di Lenola, D., et al. (2018). The association between migraine and physical exercise. The Journal of Headache and Pain, 19(1), 83.

Arruza, J.A., Arribas, S., Gil De Montes, L., Irazusta, S., Romero, S., y Cecchini, J.A. (2008). Repercusiones de la duración de la Actividad Físico-deportiva sobre el bienestar psicológico. Revista Internacional de Medicina y Ciencias de la Actividad Física y el Deporte, 8, 171-183. Badia, X., y del Llano, J. (2000). Investigación de resultados en salud. Medicina Clínica, 114, 1-7. Banco Mundial. (2015). Estimaciones del Banco Mundial usando la razón de las Perspectivas de población de la Organización de Naciones Unidas y del Banco Mundial. Recuperado de https://datos.bancomundial.org/indicator/SP.POP.65UP.FE.IN?locations=ES\&view=chart

Bassuk, S.S., y Manson, J.E. (2005). Epidemiological evidence for the role of physical activity in reducing risk of type 2 diabetes and cardiovascular disease. Journal of Applied Physiology, 99, 1193-1194.

Bennett, E., Clarke, L., Kowalski, K., y Crocker, P. (2017). From pleasure and pride to the fear of decline: Exploring the emotions in older women's physical activity narratives. Psychology of Sport Exercise, 33,113-122.

Bize, R., Johnson, J.A., y Plotnikoff, R.C. (2007). Physical activity level and health-related quality of life in the general adult population: a systematic review. Prevention Medicine, 45, 401-415.

Borg, G. (1998). Borg's perceived exertion and pain scales. Champaign, IL: Human Kinetics.

(C) Psy, Soc, \& Educ, 2020, Vol. 12(2) 
Candel, N., Olmedilla, A., y Blas, A. (2008). Relaciones entre la práctica de actividad física y el autoconcepto, la ansiedad y la depresión en chicas adolescentes. Cuadernos de Psicología del Deporte, 1, 61-77.

Capdevilla, L. (2005). Actividadfísica y estilo de vida saludable. Girona: Documenta Universitaria. Cohen, J.A. (1992). A power primer. Psychology Bulletin, 112, 155-159.

Corona-Verdú, R., y Alameda-Gadea, A. (2018). Potencia, capacidad funcional y fuerza en mujeres sanas mayores. Retos y perspectivas en el seno de una sociedad demográficamente envejecida. Prisma Social: Revista de Investigación Social. 21, 338-55.

Costa, J. (2009). Percepção dos factores ambientais e atividade física em idosos. Estudo comparativo em idosos residentes num Concelho de litoral (Esposende) e num Concelho de interior (Vila Verde) (Tesis Doctoral). Faculdade de Desporto da Universidade do Porto, Portugal.

Fortuno, J. (2011). Medida de la capacidad funcional y la calidad de vida relacionada con la salud en un grupo de personas mayores que llevan a cabo un programa de caminatas: estudio piloto. Revista Española de Geriatría y Gerontología, 46, 147-150.

Goodpaster, B.H., Park, S.W., Harris, T. B., Kritchevsky, S.B., Nevitt, M., Schwartz, A. V., ... Newman A.B. (2006). The loss of skeletal muscle strength, mass, and quality in older adults: the health, againg and body composition study. Journal of Gerontoly: Medical Science, 10, 1059-1064.

Gordon, B.R., McDowell, C.P., Lyons, M., y Herring, M.P. (2017). The Effects of Resistance Exercise Training on Anxiety: A Meta-Analysis and Meta-Regression Analysis of Randomized Controlled Trials. Sport Medicine, 47, 2521-2532.

Herrera, E., Pablos, A., Chiva-Bartoll, O., y Pablos, C. (2017). Efectos de la actividad física sobre la salud percibida y condición física de los adultos mayores. Journal of Sport \& Health Research, 9, 27-39.

Holle, V.V., Van Cauwenberg, J., Gheysen, F., Van Dyck, D., Deforche, B., Van De Weghe, N., et al. (2016). The association between Belgian older adults' physical functioning and physical activity: What is the moderating role of the physical environment? PLoS ONE, 11(2), e0148398.

Imayama, I., Alfano, C.M., Mason, C.E., Wang, C., Xiao, L., Duggan, C., ...McTiernan, A. (2013). Exercise Adherence, Cardiopulmonary Fitness, and Anthropometric Changes Improve Exercise Self-Efficacy and Health-Related Quality of Life. Journal of Physical Activity and Health, 10, 676-689.

Kanning, M., y Schlicht, W. (2008). A biopsycho-social model of successful aging as shown through the variable «physical activity». European Review of Aging and Physical Activity, 5, 79-87.

Lok, N., Lok, S., y Canbaz, M. (2017). The effect of physical activity on depressive symptoms and quality of life among elderly nursing home residents: Randomized controlled trial. Archives of Gerontology and Geriatrics, 70, 92-98.

Mannerkorpi, K., Svantesson, U., y Broberg, C. (2006). Relationships between performancebased tests and patients' ratings of activity limitations, self-efficacy, and pain in fibromyalgia. Archives of Physical Medicine and Rehabilitation, 87(2), 259-64. 
Marshall, P.W.M., Schabrun, S., y Knox, M.F. (2017). Physical activity and the mediating effect of fear, depression, anxiety, and catastrophizing on pain related disability in people with chronic low back pain. PLoS One, 12(7), e0180788.

McLean, S.A., Paxton, S.J., y Wertheim, E.H. (2010). Factors associated with body dissatisfaction and disordered eating in women in midlife. International Journal of Eating Disorders, 43(6), 527-536.

Melov, S., Tarnopolsky, M.A., Beckman, K., Felkey, K. y Hubbard, A. (2007). Resistance Exercise Reverses Aging in Human Skeletal Muscle. PLoS ONE, 2(5), e465.

Montero, I., y León, O.G. (2007). A guide for naming research studies in Psychology. International Journal of Clinical and Health Psychology, 7, 847-862.

Nelson, M., Rejeski, W.J., Blair, S.N., Duncan, P., Judge, J., King, A., ...Castaneda-Sceppa, C. (2007). Physical Activity and Public Health in Older Adults. Recommendation from the American College of Sports Medicine and the American Heart Association. Circulation, 116, 1094-1105.

Organización Mundial de la Salud [OMS]. (2002). Active ageing: a policy framework. Recuperado de https://apps.who.int/iris/bitstream/handle/10665/67215/WHO_NMH_ NPH_02.8.pdf?sequence $=1$

Pérez, B. (2014). Salud: entre la actividad física y el sedentarismo. Anales Venezolanos de Nutrición, 27, 119-128.

Phillips, S.M., Wójcicki, T.R., y McAuley, E. (2013). Physical activity and quality of life in older adults: an-18-month panel analysis. Quality of Life Research, 22, 1647-1654.

Preacher, K.J., y Hayes, A.F. (2008). Asymptotic and resampling strategies for assessing and comparing indirect effects in multiple mediator models. Behavior Research Methods, 40, 879-891.

Rehfeld, K., Müller, P., Aye, N., Schmicker, M., Dordevic, M.Kaufmann, J., ...Müller, N.G. (2017). Dancing or Fitness Sport? The Effects of Two Training Programs on Hippocampal Plasticity and Balance Abilities in Healthy Seniors. Frontiers in Human Neuroscience, 11,305 .

Rikli, R., y Jones, J. (2001). Senior Fitness Test Manual. California: Editorial Human Kinetics. Robine, J.M., y Beluche, I. (2015). European Health and Life Expectancy Information System (2015). Health Expectancy in Spain. Recuperado de http://www.eurohex.eu/pdf/ CountryReports_Issue9/Spain_Issue9.pdf

Rodrigues, L., Bherer, L., Bosquet, L., Vrinceanu, T., Nadeau, S., Lehr, L. et al. (2020). Effects of an 8-week training cessation period on cognition and functional capacity in older adults. Experimental Gerontology, 134, 110890.

Rodríguez, M., Molina, J., Jiménez, C., y Pinzón, R. (2011). Calidad de vida y actividad física en estudiantes, docentes y administrativos de una universidad de Bogotá. Cuadernos Hispanoamericanos de Psicología, 11, 19-37.

Ruiz-Montero, P.J., Castillo-Rodríguez, A., Mikalacki, M., y Delgado-Fernández, M. (2015). Physical fitness comparison and quality of life between Spanish-Serbian elderly women through a physical fitness program. Collegium Antropologicum, 39(2), 411-417.

(C) Psy, Soc, \& Educ, 2020, Vol. 12(2) 
Ruiz-Montero, P.J., Ruiz-Rico Ruiz, G.J., Martín-Moya, R., y González-Matarín, P.J. (2019). Do health-related quality of life and pain-coping strategies explain the relationship between older women participants in a pilates-aerobic program and bodily pain? A multiple mediation model. International Journal of Environmental Research and Public Health, 16(18), E3249.

Seguin, R.A., Eldridge, G., Lynch, W., y Paul, L.C. (2013). Strength Training Improves Body Image and Physical Activity Behaviors Among Midlife and Older Rural Women. Journal of Extension, 51(4), 4FEA2.

Silva, P., Rocha, L., Queirós, P., Novais, C., Botelho-Gomes, P., y Carvalho, J. (2015). Ageing and Gender: Functionality and Body's Perceptions of Older Women Submitted to an Exercise Program. Journal of Sport and Health Research, 7, 91-102.

Silva, R., y Mayán, J.M. (2016). Beneficios psicológicos de un programa proactivo de ejercicio físico para personas mayores. Escritos de Psicología, 9, 24-32.

Stillman, C.M., Cohen, J., Lehman, M.E. y Erickson, K.I. (2016). Mediators of physical activity on neurocognitive function: a review at multiple levels of analysis. Frontiers in Human Neurosciente, 10, 626.

Varoa, J.E., y Martínez-González, M.A. (2007). Current Challenges in the Research About Physical Activity and Sedentary Lifestyles. Revista Española de Cardiología, 60, 231-233. Warner, C.B., Roberts, A.R., Jeanblanc, A.B., y Adams, K.B. (2017). Coping Resources, Loneliness, and Depressive Symptoms of Older Women With Chronic Illness. Journal of Applied Gerontology, January 12, 295-322.

WHOQOL Group. (1998). The World Health Organization Quality of Life Assessment (WHOQOL): Development and general psychometric properties. Social Science Medicine, $46,1569-1585$.

Zarshenas, S., Houshvar, P., y Tahmasebi, A. (2013). The effect of short-term aerobic exercise on depression and body image in Iranian women. Depression Research and Treatment, 2013, 132684. 